\title{
Descrição Preliminar do Corpus DANTEStocks: Diretrizes de Segmentação para Anotação segundo Universal Dependencies
}

\author{
Ariani Di Felippo ${ }^{1}$, Caroline Postali ${ }^{1}$, Gabriel Ceregatto ${ }^{1}$, Laura S. Gazana ${ }^{1}$, \\ Emanuel H. da Silva ${ }^{2}$, Norton T. Roman ${ }^{3}$, Thiago A. S. Pardo ${ }^{2}$ \\ ${ }^{1}$ Núcleo Interinstitucional de Linguística Computacional (NILC) \\ Departamento de Letras - Universidade Federal de São Carlos (UFSCar) \\ Caixa Postal 676 - 13565-905 - São Carlos - SP - Brasil \\ ${ }^{2}$ Núcleo Interinstitucional de Linguística Computacional (NILC) \\ Instituto de Ciências Matemáticas e de Computação - Universidade de São Paulo (USP) \\ Caixa Postal 668 - 13566-970 - São Carlos - SP - Brazil \\ ${ }^{3}$ Escola de Artes, Ciências e Humanidades - Universidade de São Paulo (USP) \\ gabriel@ceregatto.admin.br, lauragazana@estudante.ufscar.br, \\ \{emanuel.huber, norton\}@usp.br, taspardo@icmc.usp.br
}

\begin{abstract}
The annotation of informal texts within the Universal Dependencies framework requires two segmentation processes: definition of the relevant unity for syntactic analysis and identification of syntactic words. In this paper, we present the linguistic idiosyncrasies of DANTEStocks, a corpus of tweets from the financial market, written in Portuguese, and the general guidelines for their automatic segmentation. As such, this work contributes to a better understanding of linguistic aspects of tweets and the development of resources and tools for automatic processing of this subgenre of user-generated content.
\end{abstract}

Resumo. A anotação de textos informais segundo a Universal Dependencies requer dois processos de segmentação: delimitação da unidade relevante para a análise sintática e identificação das palavras sintáticas. Neste artigo, apresentam-se as idiossincrasias linguísticas do corpus DANTEStocks, composto por tweets do mercado financeiro, escritos em Português, e as estratégias gerais de segmentação automática. Assim, contribui-se para a descrição de aspectos linguísticos dos tweets e para o desenvolvimento de recursos e ferramentas de processamento automático desse subgênero de "user-generated content".

\section{Introdução}

Diante da imensa relevância adquirida na última década, as redes sociais (como Facebook, WhatsApp, Twitter, etc.) são fontes de conteúdo (em inglês, user-generated content - UGC) inestimáveis para consumidores, políticos e governos no geral. Com isso, o desenvolvimento de ferramentas e aplicações linguístico-computacionais (como as de análise de sentimento e mineração de opinião) tem se tornado tópico central do Processamento Automático das Línguas Naturais (PLN) [Sanguinetti et al., 2020a]. 
Nesse cenário, já há vários taggers (etiquetadores morfossintáticos) [p.ex.: Owoputi et al., 2013; Lynn et al., 2015; Bosco et al., 2016; Proisl, 2018] e parsers (analisadores sintáticos) [p.ex.: Foster, 2010; Petrov, Mcdonald, 2012; Kong et al., 2014 e Liu et al., 2018] relativamente precisos para o processamento de UGCs, sobretudo em inglês. E esse ferramental só foi desenvolvido graças aos corpora anotados (treebanks) e aos algoritmos de aprendizado de máquina. Grande parte dos treebanks de UGC construídos nos últimos anos são compostos exclusivamente por tweets. O destaque dos corpora de tweets (os tweebanks) se deve pela facilidade de obtenção dos dados, política do Twitter sobre o uso dos dados para fins acadêmicos e relevância para aplicações de PLN. O tamanho desses recursos varia de 500 a aproximadamente 6,700 mensagens [Sanguinetti et al., 2020a].

Os tweebanks mais recentes possuem anotação segundo a Universal Dependencies (UD) [Nivre, 2015; Nivre et al., 2020], um modelo gramatical que fornece principalmente um conjunto de etiquetas morfossintáticas universais e de relações de dependências sintáticas para anotação de corpus, o que possibilita estudos "cross-linguísticos" e reuso de metodologias.

A anotação UD de UGC, como os tweets, requer inicialmente que a unidade relevante para a análise sintática seja definida. Isso significa decidir se essa unidade será delimitada com base na noção de sentença (como nos textos formais) ou outro critério. Ademais, por se basear em uma visão lexicalista da sintaxe, a anotação UD necessita que as palavras sintáticas ${ }^{1}$ sejam identificadas (tokenizadas) ${ }^{2}$. Para tanto, é preciso descrever as características linguísticas (estruturais, ortográficas e lexicais) do tweets que compõem o corpus que será anotado [Liu et al., 2018; Sanguinetti et al., 2020a,b]. Por exemplo, uma característica geral dos tweets é a ocorrência de autocensuras ("m*" $\rightarrow$ "merda"). Para o reconhecimento das autocensuras como palavras, é preciso prever que o asterisco não seja segmentado, mas reconhecido como parte constitutiva do token.

Neste artigo, descrevem-se as características linguísticas do corpus construído por Silva et al. (2020) e as decorrentes estratégias automáticas de segmentação (isto é, delimitação da unidade de análise sintática e tokenização) para anotação UD. Denominado DANTEStocks, o corpus é composto por tweets em português sobre ações do índice Ibovespa e possui anotação de emoções. O DANTEStocks será o primeiro corpus de UGC em português com anotação UD. Acredita-se que a anotação UD poderá potencializar o emprego do corpus nas investigações sobre análise de sentimentos e ampliar a sua utilidade em outros tipos de pesquisas linguístico-computacionais. Dessa forma, esse trabalho contribui para os estudos descritivos sobre as características linguísticas dos tweets e para o desenvolvimento de recursos, ferramentas e aplicações de processamento automático desse tipo particular de UGC.

Nas Seções 2, descreve-se brevemente o modelo UD. Na Seção 3, apresentam-se o corpus DANTEStocks, as características estruturais de seus tweets e a decorrente delimitação da unidade de análise sintática. Na Seção 4, sistematizam-se os dispositivos linguísticos (lexicais e ortográficos) que caracterizam o corpus e discute-se a tokenização de alguns deles. Na Seção 5, apresentam-se as considerações finais sobre o trabalho, destacando suas contribuições e estudos futuros.

\footnotetext{
${ }^{1}$ Palavra sintática (em inglês, syntactic word) é a unidade mínima a que corresponde uma função sintática (https://universaldependencies.org/u/overview/tokenization.html).

${ }^{2} \mathrm{Na}$ anotação UD, palavras sintáticas (ou itens lexicais) são sinônimos de tokens.
} 


\section{O Modelo Universal Dependencies}

O modelo UD prevê anotação no nível sentencial e diretrizes para tokenização e anotação morfossintática e sintática ${ }^{3}$. Sobre a tokenização, a UD, a partir de uma visão lexicalista da sintaxe, define que uma relação de dependência (deprel) ocorre entre palavras de uma sentença e as características morfológicas são representadas por propriedades (ou features). Assim, as unidades básicas de anotação são palavras sintáticas. Com isso, os clíticos precisam ser separados de seus hospedeiros ("preparese" $\rightarrow$ "prepare" "se") e tratados como palavras independentes, assim como as contrações precisam ser decompostas ("das" $\rightarrow$ "de" "as"). Excepcionalmente, o modelo permite a combinação de tokens ortográficos em uma única palavra, como é o caso das abreviações (p.ex.: “e.g."). Quanto à anotação linguística, a UD prevê 2 níveis. No nível morfológico, especificam-se 3 tipos de informação: lema, etiqueta morfossintática e traços lexicais/gramaticais (das palavras). No nível sintático, parte-se da premissa de que as deprels são relações binárias e assimétricas [Nivre, 2015; Nivre et al., 2020; Marnefee et al., 2021] e que a representação básica de uma estrutura de dependências é arbórea, na qual uma palavra é o root (raiz) da sentença.

Na Figura 1, ilustra-se a anotação UD de uma sentença de um corpus jornalístico em português. Em caixa alta, estão codificadas as etiquetas morfossintáticas, como DET para "esse", NOUN para "carro" e VERB para "achado". A versão 2.0 $0^{4}$ da UD dispõe de 17 etiquetas, juntamente com critérios para o emprego de cada uma delas. Logo acima das etiquetas, estão as formas canônicas, por exemplo: "esse", "carro" e "achar" são respectivamente os lemas de "esse", "carro" e "achado". As deprels estão indicadas por setas rotuladas que se originam no head (cabeça) e se destinam ao dependente. $\mathrm{Na}$ Figura 1, "carro", por exemplo, é dependente de "achado" (cabeça) e estes estão conectados pela deprel nsubj:pass (sujeito nominal da passiva). O verbo "achado" é a raiz da sentença-exemplo. A UD (2.0) fornece 37 relações, juntamente com critérios para o emprego de cada uma delas. A UD também fornece uma lista bastante extensa de traços que codificam propriedades lexicais e gramaticais das palavras. Embora ausentes na Figura 1, "carro", no caso, possui os traços-valores: Gender=Masc e Number=Sing 5 .

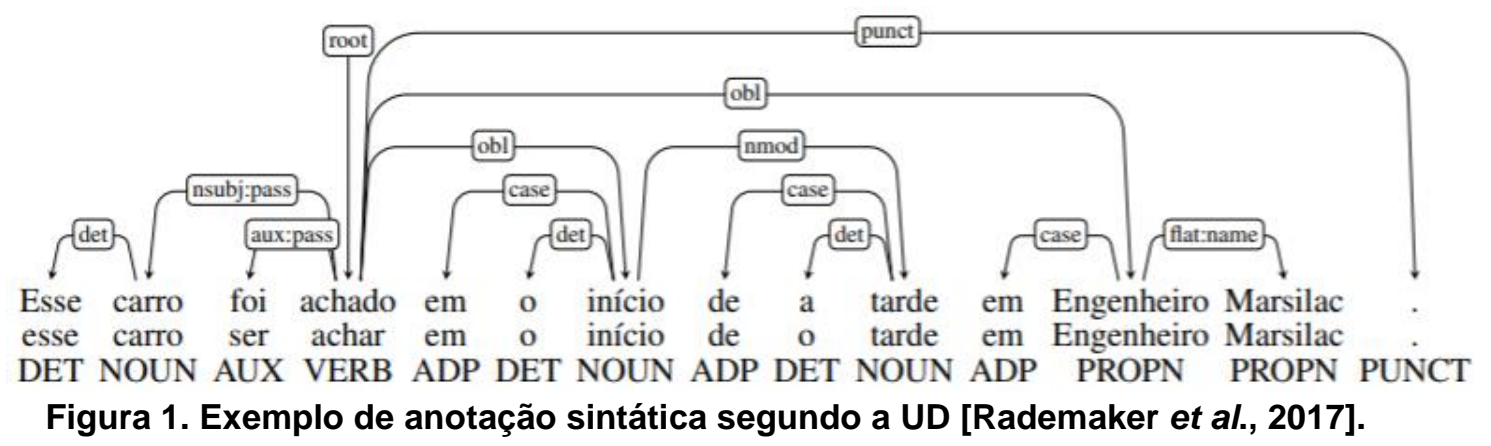

A seguir, apresenta-se o DANTEStockes para, na próxima seção, descrever as suas particularidades lexicais, ortográficas e estruturais.

\footnotetext{
${ }^{3}$ Para o português do Brasil, Duran (2021) construiu um manual com diretrizes de tokenização e de anotação morfossintática segundo a UD (especificamente para textos formais, como os jornalísticos).

${ }^{4}$ https://universaldependencies.org/guidelines.html

${ }^{5}$ Essa informação foi recuperada do corpus UD-Portuguese-Bosque por meio da plataforma online Grewmatch (http://match.grew.fr/?corpus=UD_Portuguese-Bosque@ 2.8).
} 


\section{DANTEStocks - Estrutura dos Tweets e Definição da Unidade de Análise}

O DANTEStocks ${ }^{6}$ é um corpus de material textual compilado do Twitter, que parece uma mescla de rede social e microblog ${ }^{7}$ [Freitas, Barth, 2015], e cujas principais características são a dinamicidade das interações (sejam comentários ou republicações) e a brevidade das mensagens (restrição de 140 caracteres). Considerado um gênero, o tweet parece ser constituído por resquícios de outros gêneros (como notícia, propaganda, bilhete, diário íntimo, etc.), que foram modificados para atender às necessidades de comunicação da rede [Marcuschi, 2008, Freitas, Barth, 2015]. Aliás, esses diferentes gêneros que se entrelaçam nos tweets evidenciam a influência da oralidade nessa escrita online. O DANTEStocks engloba especificamente 4.517 tweets contendo menção a alguma das ações do índice Ibovespa ${ }^{8}$. As postagens foram coletadas automaticamente em 2014 com base nos tickers (códigos) (isto é, cadeias de 4 letras e 1 número que fazem alusão ao nome da empresa e ao tipo de ação, como "PETR4") das ações do índice. O corpus, originalmente construído para pesquisas sobre análise de sentimentos, já possui anotação de emoções [cf. Silva et al., 2020].

Quanto à composição estrutural, os tweets do corpus variam bastante. Há tweets formados por uma ou mais sentenças claramente delimitadas, como (1), (2) e (3). Mas há também tweets que apresentam, frente às normas da língua padrão, ausência de pontuação (4) ou pontuação equivocada (5). Tweets relativamente fragmentados (6) também compõem o corpus. Em (4), o tweet parece ser composto por duas sentenças ("O \#PT conseguiu fazer propaganda eleitoral antecipada" e "O que a @ dilmabr tem a dizer sobre isso"). Essa interpretação pode ser corroborada pela capitalização do segundo "o" (negrito). Em (5), o exemplo é de uso inadequado da vírgula, provavelmente em substituição ao ponto de exclamação. O tweet (6) exemplifica uma postagem relativamente fragmentada, composta por uma hashtag seguida por um sintagma nominal e um link. O tweet (3), em especial, apresenta alternância de código linguístico (em inglês, code-switching) (português-inglês) em nível sentencial.

(1) Sera k petr4 já entrou na baixa?

(2) PETR4 subiu na bolsa 13,50. Muito bem, surpreso com o resultado.

(3) \#CSNA3: Está em região de suporte que vem resistindo. Who knows?

(4) O \#PT conseguiu fazer propaganda eleitoral antecipada $\mathbf{O}$ que a @dilmabr tem a dizer sobre isso?

(5) Bom dia Marcos, Alguma previsão para petr4?!

(6) \#GGBR4 Suportes e resistências http://t.co/Azw6yIEVI9

Para a anotação sintática de textos formais, a unidade de anotação é comumente a sentença, cuja segmentação automática é tarefa relativamente simples, pois a pontuação pode ser usada como critério [Reynar e Ratnaparkhi, 1997]. Buscando estabelecer certa compatibilidade com os treebanks de textos formais, Sanguinetti et al. (2020b) optam por segmentar (automaticamente) somente os tweets com sentenças bem delimitadas (como (1), (2) e (3)), podendo utilizar índices para reconstruir, se necessário, as mensagens segmentadas [Rehbein et al., 2019].

\footnotetext{
${ }^{6}$ Disponível em: https://www.kaggle.com/fernandojvdasilva/stock-tweets-ptbr-emotions/data.

${ }^{7}$ Microblog é um tipo de blog no qual os usuários fazem atualizações breves de texto (até 200 caracteres), sobretudo veiculando impressões pessoais.

${ }^{8}$ Principal índice da bolsa de valores oficial do Brasil, a B3 (de "Brasil, Bolsa, Balcão").
} 
Em outros trabalhos, o tweet é considerado unidade mínima de anotação [Kong et al., 2014; Liu et al., 2018; Sanguinetti et al., 2018]. Embora a não-segmentação dos tweets em unidades menores possa levar a um emprego excessivo da parataxis ${ }^{9}$ [Sanguinetti et al., 2020], que é a deprel usada para relacionar elementos justapostos que não estejam coordenados, subordinados ou em outra relação argumento-predicado, essa foi a opção adotada para o DANTEStocks. Com isso, a anotação UD (morfossintática, por enquanto) do corpus está sendo feita no nível do tweet.

Essa opção se justifica por algumas razões. Uma delas são os problemas de pontuação, que dificultam a segmentação sentencial automática. Embora haja outros critérios para essa segmentação, como a detecção de estruturas verbo-argumento, estes não foram considerados devido à complexidade de se processar automaticamente os tweets. Assim, considerar o tweet como unidade única economiza o esforço necessário para desenvolver, manter, adaptar ou realizar o pós-processamento em um segmentador automático. Além disso, considerar o tweet como unidade mínima pode ser relevante para pesquisas linguístico-computacionais a respeito desse tipo de UGC. Cignarella et al. (2019), por exemplo, destacam que o estudo da correlação entre aspectos sintáticos (via UD) e ironia só foi possível diante dos tweets enquanto unidade. Outra razão importante é a interpretação (e consequente anotação sintática) dos tweets, que, muitas vezes, depende da mensagem completa. Isso fica evidente diante dos tweets que têm certa fragmentação, como (6). Somente é possível interpretar que os níveis de "suporte" e "resistência" (isto é, conceitos de análise gráfica) de interesse são os relativos à ação/ticker "GGBR4" com base na mensagem completa. A anotação intersentencial de alternância de código também pode ser considerada mais apropriada no nível do tweet.

\section{Os Fenômenos UGC do DANTEStocks e a sua Tokenização}

A partir de trabalhos como os de Lyddy et al. (2014), Liu et al. (2018) e Sanguinetti et al., 2020a,b), as particularidades ortográficas e lexicais do corpus foram sistematizadas em 7 dimensões. As dimensões e os fenômenos estão exemplificados no Quadro 1.

1. Simplificação de código: engloba os fenômenos "ergográficos" (em inglês, ergographic phenomena), que reduzem o esforço de escrita de um único token, como remoção/adição de diacrítico, ausência de hífen, substituição de diacrítico (pela letra "h"), omissão de letras (finais e mediais), erro ortográfico/digitação e fonetização.

2. Abreviação: toda sequência de caracteres que representa de forma reduzida várias palavras; a abreviação pode ser do tipo contração (de elementos gramaticais), acrônimo ou inicialismo (do inglês, initialism) (isto é, abreviações compostas pelas letras iniciais de palavras comuns ("lp" $\rightarrow$ "longo prazo") [Lyddy et al., 2014].

3. Expressão de sentimento: fenômenos que emulam o sentimento expresso pela prosódia, expressão facial ou gesto na interação via tweet, como alongamento grafêmico (sobretudo de vogais), repetição de pontuação, autocensura e emoticons.

4. Influência de língua estrangeira: vocábulo formado com base em outra língua; "estopar", por exemplo, baseia-se no verbo em inglês "stop" ("parar") (isto é, interromper venda ou compra de um ativo diante de dado preço).

5. Expressão de oralidade: toda palavra cuja grafia remonta à comunicação (fala) informal, as quais são, por vezes, empregadas com função humorística.

\footnotetext{
${ }^{9}$ https://universaldependencies.org/u/dep/parataxis.html
} 
6. Elemento metalinguístico: todo elemento que tipicamente ocorre no Twitter, como hashtag, menção, marca de retweet, URL e truncamento lexical (quebra de palavra).

7. Fenômeno de domínio: todo fenômeno lexical/gráfico que diferencia os tweets do DANTEStocks dos demais tweets, a saber: tickers, cashtag, numerais com parte decimal indeterminada, índices de (des)valorização das ações, substituições lexicais (por símbolo), expressões temporais alfanuméricas e valor monetário aglutinado.

Quadro 1. Exemplo dos fenômenos UGC no DANTEStocks.

\begin{tabular}{|c|c|c|}
\hline \\
\hline Fenômeno & Exemplo & Forma padrão/glosa ${ }^{10}$ \\
\hline \multicolumn{3}{|c|}{ Simplificação de código } \\
\hline Ausência/adição de diacrítico & proprio, milhao, Graca, fêz & próprio, bilhão, Graça, fez \\
\hline Ausência de hífen & sexta feira, caça níquel & sexta-feira, caça-níquel \\
\hline Substituição de diacrítico & eh, neh, tou & $e ́, n e ́, t \hat{o}$ \\
\hline Omissão de letras & d, n, qdo, tx, ult, pq & de, não, quando, taxa, último, porque \\
\hline Erro ortográfico/digitação & comrpa, agradeveis & compra, agradáveis \\
\hline Fonetização & k, kd, krk, kct & que, cadê, caraca, cacete \\
\hline \multicolumn{3}{|c|}{ Abreviação } \\
\hline Contração & oq, pq & o que, por que, por favor \\
\hline Acrônimo/inicialismo & BB, cf, lp & Banco do Brasil, conselho fiscal, longo prazo \\
\hline \multicolumn{3}{|c|}{ Expressão de sentimento } \\
\hline Alongamento de pontuação & Onde a \#OIBR4 vai parar??? & Onde a \#OIBR4 vai parar? \\
\hline Alongamento grafêmico & noosaaa, LINNDA & nossa, linda \\
\hline Autocensura & p**a $\mathbf{m} *$ & puta, merda \\
\hline Emoticon & o.O :) :/ & surpresa, sorriso (feliz), indecisão \\
\hline \multicolumn{3}{|c|}{ Influência de língua estrangeira } \\
\hline Formação verbal & estopar & 'parar investimento' \\
\hline \multicolumn{3}{|c|}{ Marca de oralidade } \\
\hline Coloquialismo & guvêrno, bão, ae, péra, vamu & governo, bom, aí, espere, vamos \\
\hline Expressão cristalizada & né, daí (dae) & 'não é', 'de aî' \\
\hline Exclamação onomatopeica & hahaha, hehehe & risos \\
\hline \multicolumn{3}{|c|}{ Elementos metalinguísticos (do Twitter) } \\
\hline Hashtag & \#Petr4 & 'indexadores de tópicos ou assuntos' \\
\hline Menção & @ garimpodeacoes & 'perfil/usuário' \\
\hline Marca de retweet & RT @Ary_AntiPT & 'republicação de um tweet' \\
\hline URL & http://t.co/sROpyWPbIN & 'endereço da web' \\
\hline Truncamento (lexical) & Ação sobe fo... & Ação sobe fo(rte) \\
\hline \multicolumn{3}{|c|}{ Fenômeno do domínio (Ibovespa) } \\
\hline Ticker & Petr4 & 'código de uma ação' \\
\hline Cashtag & \$LREN3 & 'código de ação precedido por \$' \\
\hline Indeterminação da parte decimal & De $18, \mathbf{x x}$ a 21,00 & 'qualquer valor na parte decimal' \\
\hline Índice de (des)valorização & $+2,09 \%,-11,42 \%$ & 'percentual de (des)valorização de ação' \\
\hline Substituição lexical & ... precisam de muito $\$$ & ... precisam de muito dinheiro \\
\hline Expressão (temporal) híbrida & $1 T 14$ & primeiro trimestre de 2014 \\
\hline Valor monetário aglutinado & $\mathbf{R} \$ 20,00$ & $R \$ 20,00$ \\
\hline
\end{tabular}

${ }^{10}$ As formas de superfície do corpus não foram substituídas pelas formas da linguagem padrão, as quais estão no Quadro 1 apenas como recurso didático fornecido ao leitor para a compreensão dos fenômenos. 
Partindo-se da decisão de não normalizar os tweets do corpus com o objetivo de desenvolver ferramentas e sistemas para o mundo real, foi necessário definir o estatuto de palavra de alguns dos fenômenos sistematizados para a subsequente tokenização.

Quanto aos fenômenos de simplificação de código, ressalta-se que um composto hifenizado (como "caça-níquel") constitui, segundo a visão lexicalista da UD, uma única palavra. Assim, mesmo que a ausência do hífen, como em "caça níquel”, resulte na identificação automática de dois tokens ("caça" e "níquel"), a anotação UD precisa evidenciar que se trata de um composto, isto é, token único. Uma alternativa pode ser a utilização da deprel compound, como é feito no corpus UD_English-EWT ${ }^{11}$ em inglês.

As contrações são formas abreviadas de duas palavras funcionais com remoção de espaços e letras. Nessa categoria, no entanto, há diferentes fenômenos de redução, os quais necessitam, por isso, de estratégias distintas de tokenização. A forma superficial "oq" (em "Oq faz?"), por ser constituída por dois pronomes ("o" "que") e ter a função única de pronome, corresponde a um token único. Já "pq", ao reduzir duas palavras ("por" "que"), de categorias morfossintáticas diferentes (preposição e pronome, respectivamente), deve ser decomposta em dois tokens. Os outros tipos de abreviação, ou seja, acrônimos (que reduzem nomes de entidades), como "BB" ("Banco do Brasil"), e inicialismos (que abreviam expressões compostas por palavras comuns), como "cf" ("conselho fiscal"), são tokens únicos, uma vez que desempenham função sintática específica, sendo possível atribuir à forma reduzida a categoria morfossintática do head.

Quanto às expressões de sentimento, destaca-se que as autocensuras, como "car*" ("caralho"), e os emoticons (";--*" $\rightarrow$ "beijo") correspondem a palavras sintáticas. No entanto, o adequado reconhecimento destes como tal requer que os sinais de pontuação e os caracteres especiais sejam reconhecidos como elementos constitutivos do token. No DANTEStocks, os emoticons ocorrem ao final dos tweets, não havendo uma ligação clara com a estrutura do tweet, a não ser "discursiva".

As marcas de oralidade classificadas como "expressão cristalizada" - "né" e "daí" (ou "dae") - são etimologicamente contrações de "não é" e "de aí". Sendo contrações (ou seja, tokens compostos por mais de uma categoria gramatical), elas seriam tokenizadas segundo a UD. No entanto, essas expressões funcionam no corpus como uma unidade, sendo o mais adequado, nesse caso, não realizar a decomposição. Atualmente, a categoria gramatical mais adequada a ser atribuída a elas está sob estudo (se advérbio ou interjeição). No nível sintático, no entanto, sabe-se que essas expressões desempenham função discursiva e a anotação via deprel precisará evidenciar isso.

Sobre os elementos metalinguísticos, os truncamentos lexicais ocorrem principalmente no fim de um tweet devido ao limite de caracteres. Na literatura, eles são tokenizados e, caso as formas completas possam ser recuperadas, os truncamentos são anotados em função delas. No que diz respeito às hashtags (e também cashtags) e menções, o reconhecimento dos símbolos "\$" e “@”" como parte constitutiva dos tokens parece variar na literatura. No DANTEStocks, esses símbolos foram considerados como tal, compondo um token único com a palavra ou expressão que eles precedem.

Quanto aos fenômenos de domínio, os índices de (des)valorização das ações compreendem 3 tokens (“+2,09\%" $\rightarrow$ " + " "2,09" “\%”). Especificamente, reconhecer "+” como token (no caso, um símbolo) justifica-se pela possibilidade de substituí-lo por

\footnotetext{
${ }^{11}$ https://github.com/UniversalDependencies/UD_English-EWT
} 
outra palavra (como "subiu"). Outra característica de domínio são as formas reduzidas de expressões temporais, como "1T14" ("primeiro trimestre de 2014"). Estas, ao funcionarem como unidade, são consideradas palavras únicas e anotadas com a categoria morfossintática do head, como sugerido para os acrônimos e inicialismos. No DANTEStocks, as expressões monetárias podem ocorrer aglutinadas (isto é, sem espaço entre o símbolo monetário e o numeral) ("R \$20,00"). Estas, no entanto, são compostas por dois tokens (já que "R $\$ 20,00 "$ é o mesmo que "vinte reais") e, por isso, precisam ser tokenizadas.

\section{Considerações finais}

A caracterização linguística ora apresentada revelou que os tweets do DANTEStocks são marcados por convenções e limitações impostas pela plataforma, marcas de informalidade e certos dispositivos linguísticos, alguns deles, aliás, dependentes de domínio. $\mathrm{O}$ estudo sobre a estrutura dos tweets e a descrição dos dispositivos lexicais e gráficos fundamentaram a segmentação do corpus para a anotação UD. A definição do estatuto de token dos fenômenos resultou em algumas regras contextuais utilizadas por Silva et al. (2021) para adaptar o tokenizador simbólico de tweets do pacote NLTK $^{12}$ ao DANTEStocks. Para dar continuidade a este trabalho, pretende-se quantificar os fenômenos no corpus, gerando estatísticas de frequência/relevância.

\section{Agradecimentos}

Os autores deste trabalho agradecem ao Centro de Inteligência Artificial (C4AI - USP) e o apoio da Fundação de Apoio à Pesquisa do Estado de São Paulo (processo FAPESP \#2019/07665-4) e da IBM Corporation.

\section{Referências}

Bosco, C., Tamburini, F., Bolioli, A., Mazzei, A. (2016). Overview of the EVALITA 2016 Part of Speech tagging on TWitter for ITAlian task. In: Anas do $5^{\circ}$ EVALITA.

Cignarella, A.T., Bosco, C., Rosso, P. (2019). Presenting TWITTIRO-UD: an Italian twitter treebank in Universal Dependencies. In: Anais do $5^{\circ}$ Depling, p.190-7. Paris, França, ACL.

Duran, M.S. (2021). Manual de anotação de PoS tags. Relatório Técnico, n. 434. NILCICMC/USP, 54p. Disponível em: https://sites.google.com/icmc.usp.br/poetisa. Acesso em: 20/09/2021.

Eisenstein, J. (2013). What to do about bad language on the internet. In: Anais do NAACLHLT, p. 359-369. Atlanta, EUA, ACL.

Foster, J. (2010). "cba to check the spelling": investigating parser performance on discussion forum posts. In: Anais do NAACL-HLT, p. 381-384. LA, EUA, ACL.

Freitas, E.C.; Barth, P.A. (2015) Gênero ou suporte? O entrelaçamento de gêneros no Twitter. Revista (Con)Textos Linguísticos, 9(12), p. 08-26.

Kong, L., Schneider, N., Swayamdipta, S., Bhatia, A., Dyer, C., Smith, N.A. (2014). A dependency parser for tweets. In: Anais do EMNLP, p. 1001-12. Doha, Qatar.

Lyddy, F., Farina, F., Hanney, J., Farrell, L., O'Neill, N.K. (2014). An analysis of language in university students' text messages. Journal of Computer-Mediated Communication, 19(3), p. 546-561. Wiley Online Library.

\footnotetext{
12 https://www.nltk.org/api/nltk.tokenize.html
} 
Lynn, T., Scannell, K., Maguire, E. (2015). Minority language Twitter: part-of-speech tagging and analysis of Irish tweets. In: Anais do ACL'15 Workshop on Noisy User-generated Text, p. 1-8. July 31. Beijing, China, ACL.

Liu, Y., Zhu, Y., Che, W., Qin, B., Schneider, N., Smith, N.A. (2018). Parsing tweets into Universal Dependencies. In: Anais do NAACL-HLT, p. 965-975. LA, EUA, ACL.

Marcuschi, L.A. Produção textual, análise de gêneros e compreensão. Parábola Ed., 2008.

De Marneffe, M-C., Manning, C.D., Nivre, J. Zeman, D. (2021). Universal Dependencies. In Computational Linguistics, 47(2), p. 255-308. ACL. Online ISSN 1530-9312.

Nivre, J. (2015). Towards a Universal Grammar for Natural Language Processing. In: Anais do CICLing 2015. Lecture Notes in Computer Science, vol 9041, p. 3-16, Ed. by A. Gelbukh. Springer, Cham.

Nivre, J. et al. (2020). Universal Dependencies v2: an evergrowing multilingual treebank collection. In: Anais do $12^{\circ}$ LREC. P. 4034-4043. Marseille, França. ELRA.

Owoputi, O., O’Connor, B., Dyer, C., Gimpel, K., Schneider, N., Smith, N.A. (2013). Improved part-of-speech tagging for online conversational text with word clusters. In: Anais do NAACL-HLT, p. 380-390. 9-14 de junho. Atlanta, Georgia. ACL.

Petrov, S., Das, D., McDonald, R. (2012). A universal part-of-speech tagset. In: Anais do $8^{\circ}$ LREC, p. 2089-2096. 21-27 de maio. Istanbul, Turquia. ELRA.

Proisl, T. (2018). Someweta: A part-of-speech tagger for German social media and web texts. In: Anais do $11^{\circ}$ LREC, p. 665-670. May 7-12. Miyazaki, Japão. ELRA.

Plutchik R., Kellerman, H. (eds). 1986. Emotion: theory, research and experience. Nova Iorque: Acad. Press

Rademaker, A.; Chalub, F., Real, L., Freitas, C., Bick, E., Paiva, V. (2017). Universal Dependencies for Portuguese. In: Anais do $4^{\circ}$ Depling, p. 197-206. Pisa, Itália.

Rehbein, I., Ruppenhofer, J., Bich-Ngoc, D. (2019). tweeDe - a Universal Dependencies treebank for German tweets. In: Anais do $18^{\circ}$ TLT, p. 100-108. Paris, França. ACL.

Reynar, J., Ratnaparkhi, A. (1997). A maximum entropy approach to identifying sentence boundaries. In: Anais do $5^{\circ}$ ANLP, p. 16-19. Washington, EUA, ACL.

Seddah, D., Sagot, B., Candito, M., Mouilleron, V., Combet, V. (2012). The French social media bank: a treebank of noisy user generated content. In: Anais do $24^{\circ}$ COLING, p. 2441-2458, Mumbai, Índia, ACL.

Sanguinetti, M. et al. (2018). PoSTWITA-UD: An Italian twitter treebank in Universal Dependencies. In: Anais do $11^{\circ}$ LREC. p. 1768-75. Miyazaki, Japão. ELRA

Sanguinetti, M. et al. (2020a). Treebanking user-generated content: a proposal for a unified representation in universal dependencies. In: Anais do $12^{\circ}$ LREC. p. 5240-50. Marseille, França. ELRA

Sanguinetti, M. et al. (2020b). Treebanking user-generated contente: a UD based overview of guidelines, corpora and unified recommendations. Available in: https://arxiv.org/abs/2011.02063. Access in: 25/09/2021.

Silva, F.J.V., Roman, N.T., Carvalho, A.M.B.R. (2020). Stock market tweets annotated with emotions. In: Corpora, 15(3), p. 343-354. Online ISSN: 1755-1676.

Silva, E.H., Pardo, T.A.S., Roman. N.T, Di-Felippo, A. Universal Dependencies for tweets in Brazilian Portuguese: tokenization and part of speech tagging. In: Anais do XVIII ENIAC 2021. 29 de nov. a 3 de dez., 2021. No prelo 